\title{
CONTRACTIVE PROJECTIONS IN ORLICZ SEQUENCE SPACES
}

\author{
BEATA RANDRIANANTOANINA
}

Received 23 June 2003

We characterize norm-one complemented subspaces of Orlicz sequence spaces $\ell_{M}$ equipped with either Luxemburg or Orlicz norm, provided that the Orlicz function $M$ is sufficiently smooth and sufficiently different from the square function. We measure smoothness of $M$ using $A C^{1}$ and $A C^{2}$ classes introduced by Maleev and Troyanski in 1991, and the condition for $M$ to be different from a square function is essentially a requirement that the second derivative $M^{\prime \prime}$ of $M$ cannot have a finite nonzero limit at zero. This paper treats the real case; the complex case follows from previously known results.

\section{Introduction}

The study of norm-one projections and their ranges (one-complemented subspaces) has been an important topic of the isometric Banach space theory since the inception of the field. Contractive projections were also investigated from the approximation theory point of view, as part of the study of minimal projections, that is, projections onto the given subspace with the smallest possible norm (cf. $[5,14]$ ). They are also closely related to the metric projections or nearest point mappings, and they are a natural extension of the notion of orthogonal projections from Hilbert spaces to general Banach spaces. Despite a great amount of work on contractive projections (cf. the survey [19]), not much is known about them in Orlicz spaces.

In Lebesgue spaces $L_{p}$ and $\ell_{p}, 1 \leq p<\infty$, a subspace $Y$ is one-complemented if and only if $Y$ is isometrically isomorphic to an $L_{p}$-space of appropriate dimension $($ see $[1,6])$. This result has no analogue in other spaces. Lindberg [10] demonstrated that there exist classes of Orlicz sequence spaces $\ell_{M}$ containing one-complemented subspaces which are not even isomorphic to $\ell_{M}$. He showed that for all $1<a \leq b<\infty$, there exists a reflexive Orlicz sequence space $\ell_{M}$ so that for all $p \in[a, b]$, there is a contractive projection from $\ell_{M}$ onto a subspace isomorphic to $\ell_{p}$. This implies, in particular, that Orlicz sequence spaces can have continuum isomorphic types of one-complemented subspaces.

On the other hand, one-complemented subspaces of $\ell_{p}$ are also characterized as subspaces which are spanned by a family of mutually disjoint elements of $\ell_{p}$ (see $[2,11]$ ). Moreover, all known examples of one-complemented subspaces in symmetric Banach 
spaces with one-unconditional bases and sufficiently different from Hilbert spaces are spanned by a family of mutually disjoint vectors. (Note that, since in Hilbert spaces every subspace is one-complemented, it is necessary to include in this context some kind of an assumption about the space being different from Hilbert space.) In particular, the above-mentioned examples of Lindberg of one-complemented subspaces of Orlicz sequence spaces are spanned by mutually disjoint vectors and the norm-one projection is an averaging projection. It was shown in [16] that indeed every one-complemented subspace $Y$ in any complex Banach space $X$, with a one-unconditional basis (not necessarily symmetric) which does not contain a one-complemented isometric copy of a two-dimensional Hilbert space $\ell_{2}^{2}$, has to be spanned by a family of disjointly supported elements of $X$ and the norm-one projection from $X$ onto $Y$ has to be the averaging projection. In particular, this holds in complex Orlicz sequence spaces $\ell_{M}$ equipped with either the Luxemburg or the Orlicz norm when $M$ is sufficiently different from the square function (cf. Remark 3.5).

In the real case, this statement in its full generality is false (cf. [16]). For real spaces, we only had the following much less satisfactory result describing special one-complemented subspaces of finite codimension in Orlicz sequence spaces $\ell_{M}$.

Theorem 1.1 [17, Theorem 7]. Let $M$ be an Orlicz function such that $M$ satisfies condition $\Delta_{2}$ at zero, $M(t)>0$ for all $t>0$ and $M$ is not similar to $t^{2}$ (it is said that $M$ is similar to $t^{2}$ if there exist constants $C, t_{0}$ so that $M(t)=C t^{2}$ for all $\left.t<t_{0}\right)$. Let $\ell_{M}$ be the Orlicz space equipped with either the Luxemburg or the Orlicz norm and $F \subset \ell_{M}$ a subspace of finite codimension. If $F$ contains at least one basis vector and $F$ is one-complemented in $\ell_{M}$, then $F$ is spanned by a family of disjointly supported vectors.

In the present paper, we prove a much stronger result-we eliminate all additional assumptions on $F$. We show that when $M$ is a sufficiently smooth Orlicz function which satisfies condition $\Delta_{2}$ and is sufficiently different from the square function, then every one-complemented subspace of the real Orlicz space $\ell_{M}$ is spanned by a family of mutually disjoint vectors and every norm-one projection in $\ell_{M}$ is an averaging projection (see Theorem 3.3 and Corollary 3.4). Here we measure smoothness of $M$ using $A C^{1}$ and $A C^{2}$ classes introduced by Maleev and Troyanski [12], and the condition for $M$ to be different from a square function is essentially a requirement that the second derivative $M^{\prime \prime}$ of $M$ cannot have a finite nonzero limit at zero.

Moreover, using [16, Theorem 6.1], it follows that if, in addition, $\ell_{M}$ is not isomorphic to $\ell_{p}$ for any $p \in[1, \infty)$, then every one-complemented subspace of $\ell_{M}$ is spanned by a block basis with constant coefficients, that is, by mutually disjoint finitely supported elements $v_{j}$ in $\ell_{M}$ of the form $v_{j}=\sum_{i \in \operatorname{supp} v_{j}} \varepsilon_{i} e_{i}$, where $\varepsilon_{i}= \pm 1$ and $\left(e_{i}\right)_{i}$ is the standard basis of $\ell_{M}$.

Our results are valid in Orlicz spaces equipped with either the Luxemburg or the Orlicz norm. Our method of proof is different from that of [17]; it relies on new results characterizing averaging projections through properties related to and generalizing disjointnesspreserving operators [15].

Recently, Jamison et al. [7] obtained (using different techniques) a generalization of Theorem 1.1 in another direction-they characterized one-complemented subspaces of 
finite codimension in sufficiently smooth Musielak-Orlicz sequence spaces, whose Orlicz function is sufficiently different from the square function.

We follow standard definitions and notations as may be found in $[8,11]$. Throughout the paper, unless otherwise noted, all spaces are over $\mathbb{R}$.

\section{Preliminary definitions}

Below we recall the basic definitions and facts about Orlicz spaces that will be important for the present paper, as may be found, for example, in $[3,8,20]$.

We say that a function $M: \mathbb{R} \rightarrow[0, \infty)$ is an Orlicz function if $M$ is even, continuous, convex, $M(0)=0, M(1)=1, \lim _{u \rightarrow 0} M(u) / u=0$, and $\lim _{u \rightarrow \infty} M(u) / u=\infty$. Note that since the Orlicz function $M$ is convex, it has the right derivative $M^{\prime}$. Let $q$ be the right inverse of $M^{\prime}$ (i.e., $\left.q(s)=\sup \left\{t: M^{\prime}(t) \leq s\right\}\right)$. Then we define the complementary function of $M$ by

$$
M^{*}(v)=\int_{0}^{|v|} q(s) d s
$$

Function $M^{*}$ is also an Orlicz function.

We say that the Orlicz function $M$ satisfies the $\Delta_{2}$ condition near zero $\left(M \in \Delta_{2}\right)$ if there exist constants $k>0$ and $u_{0} \geq 0$ such that $M\left(u_{0}\right)>0$ and for all $u$ with $|u| \leq u_{0}$,

$$
M(2 u) \leq k M(u)
$$

Note that $M \in \Delta_{2}$ does not imply that $M^{*} \in \Delta_{2}$. by

The Orlicz function $M$ generates the modular defined for scalar sequences $x=\left(x_{j}\right)_{j \in \mathbb{N}}$

$$
\rho_{M}(x)=\sum_{j=1}^{\infty} M\left(x_{j}\right) .
$$

The Orlicz sequence space $\ell_{M}$ is the space of sequences $x$ such that there exists $\lambda>0$ with $\rho_{M}(\lambda x)<\infty$. If $M \in \Delta_{2}$, then $\ell_{M}=\left\{x: \rho_{M}(\lambda x)<\infty\right.$ for all $\left.\lambda \in \mathbb{R}\right\}$. The Orlicz sequence space $\ell_{M}$ is usually equipped with one of the following two equivalent norms:

(1) the Luxemburg norm defined by

$$
\|x\|_{M}=\inf \left\{\lambda: \rho_{M}\left(\frac{x}{\lambda}\right) \leq 1\right\}
$$

(2) the Orlicz norm defined by

$$
\|x\|_{M}^{O}=\sup \left\{\sum_{j=1}^{\infty} x_{j} y_{j}: \rho_{M^{*}}(y) \leq 1\right\} .
$$

If $M \in \Delta_{2}$, then these norms are dual to each other in the following sense:

$$
\begin{aligned}
& \left(\ell_{M},\|\cdot\|_{M}\right)^{*}=\left(\ell_{M^{*}},\|\cdot\|_{M^{*}}^{O}\right), \\
& \left(\ell_{M},\|\cdot\|_{M}^{O}\right)^{*}=\left(\ell_{M^{*}},\|\cdot\|_{M^{*}}\right) .
\end{aligned}
$$


We say that two Orlicz functions $M_{1}$ and $M_{2}$ are equivalent at zero if there exist $u_{0}>0$, $k, l>0$, and $C>0$ such that $M_{1}\left(u_{0}\right)>0$ and for all $u$ with $|u| \leq u_{0}$,

$$
C^{-1} M_{2}(k u) \leq M_{1}(u) \leq C M_{2}(l u)
$$

Recall that $M_{1}$ and $M_{2}$ are equivalent at zero if and only if $\ell_{M_{1}}=\ell_{M_{2}}$ (as sets) and the identity mapping is an isomorphism (in either norm) [11]. We note that if $M \in \Delta_{2}$, then every Orlicz function $M_{1}$ equivalent to $M$ also satisfies $M_{1} \in \Delta_{2}$. Krasnosel'skiu and Rutickil proved the following characterization of the $\Delta_{2}$-condition at zero in terms of the right derivative $M^{\prime}$ of $M$.

Proposition 2.1 [8, Theorem 4.1]. Let $M$ be an Orlicz function. Then $M \in \Delta_{2}$ if and only if there exist constants $\alpha$ and $u_{0} \geq 0$ such that for $0 \leq u \leq u_{0}$,

$$
\frac{u M^{\prime}(u)}{M(u)}<\alpha,
$$

where $M^{\prime}$ is the right derivative of $M$. Moreover, if (2.9) is satisfied, then $M(2 u) \leq 2^{\alpha} M(u)$ for $0 \leq u \leq u_{0} / 2$.

In [18], we introduced another condition which on one hand is very similar to (2.9), but on the other hand is in its nature of "smoothness type," as we explain below.

Definition 2.2. Let $M \in \Delta_{2}$ be a twice differentiable Orlicz function. Function $M$ satisfies condition $\Delta_{2+}$ near zero if there exist constants $\beta>0$ and $u_{0} \geq 0$ such that for all $u \leq u_{0}$,

$$
\frac{u M^{\prime \prime}(u)}{M^{\prime}(u)}<\beta
$$

As proved in [18], condition $\Delta_{2+}$ is of "smoothness type" in the following sense:

(i) for every Orlicz function $M \in \Delta_{2}$, there exists an equivalent Orlicz function $M_{1} \in$ $\Delta_{2+}$; (however, we do not know whether for every $\varepsilon>0$, it is possible to choose $M_{1}$ so that it is $(1+\varepsilon)$-equivalent with $\left.M\right)$,

(ii) for every Orlicz function $M \in \Delta_{2+}$, there exists an equivalent (even up to an arbitrary $\varepsilon>0$ ) Orlicz function $M_{1} \notin \Delta_{2+}$.

We say that a Banach space $X$ is smooth if every element $x \in X$ has a unique norming functional $x^{*} \in X^{*}$, that is, the functional with the property that $\left\|x^{*}\right\|_{X^{*}}^{2}=\|x\|_{X}^{2}=$ $x^{*}(x)$. If $M \in \Delta_{2}$, then an Orlicz space $\ell_{M}$ (with either norm) is smooth whenever $M$ is differentiable everywhere (cf. [3, 4]). Maleev and Troyanski [12] considered a stronger notion of smoothness in Orlicz spaces which guarantees the differentiability of the norm. We recall the relevant definitions and results.

Definition 2.3 (see [13], cf. [11, page 143]). To every Orlicz function $M$, the following Matuszewska-Orlicz index is associated:

$$
\alpha_{M}^{0}=\sup \left\{p: \sup \left\{\frac{M(\lambda t)}{t^{p} M(\lambda)}: \lambda, t \in(0,1]\right\}<\infty\right\} .
$$


Definition 2.4 [12]. An Orlicz function $M$ belongs to the class $A C^{k}$ at zero $\left(M \in A C^{k}\right)$ if

(i) $\alpha_{M}^{0}>k$,

(ii) $M^{(k)}$ is absolutely continuous in every finite interval,

(iii) $t^{k+1}\left|M^{(k+1)}(t)\right| \leq c M(c t)$ a.e. in $[0, \infty)$ for some $c>0$.

Definition 2.5. Let $X, Y$ be Banach spaces. The function $\varphi: X \rightarrow Y$ is said to be $k$-times differentiable at $x \in X$ if for every $j, 1 \leq j \leq k$, there exists a continuous symmetric $j$-linear form $T_{x}^{j}: X \times \cdots \times X=X^{(j)} \rightarrow Y$ so that

$$
\varphi(x+\alpha y)=\varphi(x)+\sum_{j=1}^{k} \alpha^{j} T_{x}^{j}(y, \ldots, y)+\sigma_{x}\left(|\alpha|^{k}\right)
$$

uniformly on $y$ from the unit sphere $S(X)$ of $X$.

For an open set $V \subset X, \varphi \in F^{k}(V, Y)$ means that $\varphi$ is $k$-times differentiable at every point of $V$. If (2.12) is fulfilled uniformly on $x$ over a set $W \subset V$, we will say that $\varphi$ is $k$-times uniformly differentiable over $W$ and will write $\varphi \in U F^{k}(W, Y)$. We say that $X$ is $U F^{k}$-smooth if the norm in $X$ belongs to $U F^{k}(S(X), \mathbb{R})$.

Maleev and Troyanski proved the following results.

Theorem 2.6 [12, Theorem 6]. Let $M$ be an Orlicz function such that $M \in \Delta_{2}$ and $M \in$ $A C^{k}$. Then $\ell_{M}$ equipped with the Luxemburg norm is $U F^{k}$-smooth.

Theorem 2.7 [12, Corollary 10]. Let $M$ be an Orlicz function with $M \in \Delta_{2}$. Then for every $k \in \mathbb{N}$ such that $k<\alpha_{M}^{0}$, there exists an Orlicz function $\widetilde{M}$ equivalent to $M$ at zero so that $\ell_{\widetilde{M}}$ (with the Luxemburg norm) is $U F^{k}$-smooth. (In particular, $\ell_{\widetilde{M}}$ is isomorphic to $\ell_{M}$.)

It is well known (see, e.g., [3]) that any Orlicz function $M$ can be "smoothed out," that is, for any $M$, there exists an equivalent Orlicz function $M_{1}$ such that $M_{1}$ is twice differentiable everywhere, $M_{1}^{\prime \prime}$ is continuous on $\mathbb{R}$, and $M_{1}^{\prime \prime}(u)>0$ for all $u>0$. Moreover, given any $\varepsilon>0$, it is possible to choose $M_{1}$ so that $\ell_{M}$ and $\ell_{M_{1}}$ are $(1+\varepsilon)$-isomorphic to each other [3]. However, we do not know whether in Theorem 2.7, it is possible for any $\varepsilon>0$ to select $\widetilde{M}$ so that $\ell_{M}$ and $\ell_{\widetilde{M}}$ are $(1+\varepsilon)$-isomorphic or not.

Recall that a Banach lattice $X$ is called strictly monotone if $\|x+y\|>\|x\|$ for all $x, y \geq 0$, $y \neq 0$, in $X$. An Orlicz space $\ell_{M}$ with the Luxemburg norm is strictly monotone whenever $M \in \Delta_{2}$ (cf. [9]).

Next, we recall some facts about contractive projections and about disjointness in Orlicz spaces that we will need.

If $X$ is a Banach space with a one-unconditional basis $\left\{e_{n}\right\}_{n \in \mathbb{N}}$ and $x=\sum_{n \in \mathbb{N}} x_{n} e_{n} \in X$, then the support of $x$ is defined as $\operatorname{supp} x=\left\{n \in \mathbb{N}: x_{n} \neq 0\right\}$. We say that elements $x, y \in X$ are disjoint if $\operatorname{supp} x \cap \operatorname{supp} y=\varnothing$. We say that a projection $P$ on a Banach space with a one-unconditional basis is an averaging projection if there exist mutually disjoint elements $\left\{u_{j}\right\}_{j \in J}$ in $X$ and functionals $\left\{u_{j}^{*}\right\}_{j \in J}$ in $X^{*}$ so that $u_{j}^{*}\left(u_{k}\right)=0$ if $j \neq k, u_{j}^{*}\left(u_{j}\right)=1$ for all $j \in J$, and for each $f \in X$,

$$
P f=\sum_{j \in J} u_{j}^{*}(f) u_{j}
$$


In [15], we introduced two abstract conditions relevant for the study of averaging projections. Namely, if $X$ is a Banach space with a one-unconditional basis and $P: X \rightarrow X$ is a linear operator on $X$, we say that the operator $P$ is semi-band preserving if for all $f, g \in X$,

$$
\operatorname{supp}(P f) \cap \operatorname{supp}(g)=\varnothing \Longrightarrow \operatorname{supp}(P f) \cap \operatorname{supp}(P g)=\varnothing,
$$

and we say that $P$ is semi-containment preserving if for all $f, g \in X$,

$$
\operatorname{supp} g \subset \operatorname{supp} P f \Longrightarrow \operatorname{supp} P g \subset \operatorname{supp} P f \text {. }
$$

It is clear that all averaging projections are both semi-band preserving and semicontainment preserving. In [15], we proved that in certain situations, these conditions characterize averaging projections among contractive projections. Namely, we have the following theorem.

Theorem 2.8 [15]. Let $X$ be a purely atomic strictly monotone Banach lattice and let $P: X \rightarrow X$ be a norm-one projection which is semi-band preserving or semi-containment preserving. Then $P$ is an averaging projection.

Next, we recall conditions which partially describe disjointness and containment of supports of elements in Orlicz spaces.

Proposition 2.9 [18, Proposition 3.1]. Let $M$ be an Orlicz function with $M \in \Delta_{2+}$ and such that $M^{\prime \prime}$ is continuous on $[0, \infty), M^{\prime \prime}(0)=0$, and $M^{\prime \prime}(t)>0$ for all $t>0$. Let $f, g \in \ell_{M}$ and $N(\alpha)=\|f+\alpha g\|_{M}$. Then

(a) if $f, g$ are disjoint and $\operatorname{card}(\operatorname{supp} g)<\infty$, then $N^{\prime}(0)=0$ and $N^{\prime \prime}(\alpha) \rightarrow 0$, as $\alpha \rightarrow 0$ along a subset of $[0,1]$ of full measure;

(b) if $N^{\prime}(0)=0$ and $N^{\prime \prime}(\alpha) \rightarrow 0$, as $\alpha \rightarrow 0$ along a subset of $[0,1]$ of full measure, then $f$, $g$ are disjoint.

Proposition 2.10 [18, Proposition 4.1]. Let $M$ be an Orlicz function with $M \in \Delta_{2+}$ and such that $M^{\prime \prime}$ is continuous on $(0, \infty)$ with $\lim _{t \rightarrow 0} M^{\prime \prime}(t)=\infty$. Let $f, g \in \ell_{M}$ with $f, g \neq 0$ and $N(\alpha)=\|f+\alpha g\|_{M}$. Then

(a) if $\operatorname{card}(\operatorname{supp} g \backslash \operatorname{supp} f)>0$, then $N^{\prime \prime}(\alpha) \rightarrow \infty$, as $\alpha \rightarrow 0$ along a subset of $[0,1]$ of full measure;

(b) if $g$ is simple (i.e., $\operatorname{card}(\operatorname{supp} g)<\infty)$ and $\operatorname{supp} g \subset \operatorname{supp} f$, then there exist a subset $E$ of $[0,1]$ of full measure and $C>0$ such that for all $\alpha \in E, N^{\prime \prime}(\alpha) \leq C$.

Remark 2.11. We stress that the theorems in [18] are proven for Orlicz spaces equipped with the Luxemburg norm, and the analogs of most of the results from [18] are false in Orlicz spaces equipped with the Orlicz norm.

Remark 2.12. We note here that all the theorems in [18] were formulated and proved for Orlicz function spaces $L_{M}$, where $M$ is an Orlicz function satisfying conditions $\Delta_{2}$ and $\Delta_{2+}$ near infinity. However, to adapt to the case of Orlicz sequence spaces $\ell_{M}$, where $M$ is an Orlicz function satisfying conditions $\Delta_{2}$ and $\Delta_{2+}$ near zero, the proofs require only very 
minor changes, if any. We omit the details, which are routine but require cumbersome notation.

Finally we recall a result from [16] which describes the form of two-dimensional onecomplemented subspaces of Orlicz sequence spaces when the two spanning elements have disjoint supports. This result will allow us to give a very detailed description of onecomplemented subspaces of any dimension of Orlicz sequence spaces.

Theorem 2.13 [16, Theorem 6.1]. Let $M$ be an Orlicz function with $M \in \Delta_{2}$, let $\ell_{M}$ be a (real or complex) Orlicz sequence space equipped with either the Luxemburg or the Orlicz norm, and let $x, y \in \ell_{M}$ be disjoint norm-one elements such that $\operatorname{span}\{x, y\}$ is onecomplemented in $\ell_{M}$. Then one of the following three possibilities holds:

(1) $\operatorname{card}(\operatorname{supp} x)<\infty$ and $\left|x_{i}\right|=\left|x_{j}\right|$ for all $i, j \in \operatorname{supp} x$;

(2) there exists $p \in[1, \infty)$ such that $M(t)=C t^{p}$ for all $t \leq\|x\|_{\infty}$; or

(3) there exists $p \in[1, \infty)$ and constants $C_{1}, C_{2} \geq 0$ such that $C_{2} t^{p} \leq M(t) \leq C_{1} t^{p}$ for all $t \leq\|x\|_{\infty}$ and there exists $\gamma>0$ such that, for all $j \in \operatorname{supp} x$,

$$
\left|x_{j}\right| \in\left\{\gamma^{m} \cdot\|x\|_{\infty}: m \in \mathbb{Z}\right\}
$$

We note that it follows from Theorem 2.13 that if the Orlicz space $\ell_{M}$ is not isomorphic to $\ell_{p}$ for any $p \in[1, \infty)$, then the possibility (1) has to hold. Hence every onecomplemented disjointly supported subspace of any dimension of $\ell_{M}$ needs to be spanned by mutually disjoint finitely supported elements $v_{j}$ in $\ell_{M}$ of the form $v_{j}=\sum_{i \in \operatorname{supp} v_{j}} \varepsilon_{i} e_{i}$, where $\varepsilon_{i}= \pm 1$ and $\left(e_{i}\right)_{i}$ is the standard basis of $\ell_{M}$.

\section{Main results}

We start from a key technical lemma which will allow us to apply Propositions 2.9 and 2.10 to study whether contractive projections in Orlicz sequence spaces are semi-band preserving or semi-containment preserving.

Lemma 3.1. Let $\varphi, \psi: \mathbb{R} \rightarrow[0, \infty)$ be convex functions, differentiable everywhere, and such that $\varphi(0)=\psi(0)$ and $\varphi(\alpha) \leq \psi(\alpha)$ for all $\alpha \in \mathbb{R}$. Then

(i) $\psi^{\prime}(0)=\varphi^{\prime}(0)$;

(ii) if $\varphi^{\prime \prime}(0), \psi^{\prime \prime}(0)$ exist and $\psi^{\prime \prime}(0)=0$, then $\varphi^{\prime \prime}(0)=0$;

(iii) suppose that $\varphi^{\prime}$ and $\psi^{\prime}$ are absolutely continuous on $[0,1]$ and that $\varphi^{\prime \prime}(\alpha) \rightarrow \infty$, as $\alpha \rightarrow 0$ along a subset of $[0,1]$ of full measure. Then for every $C>0$,

$$
\mu\left(\left\{\alpha \in[0,1]: \psi^{\prime \prime}(\alpha) \text { exists and } \psi^{\prime \prime}(\alpha) \leq C\right\}\right)<1
$$

Proof. To prove (i), observe that, since $\varphi(0)=\psi(0)$, we have for all $\alpha \in \mathbb{R}, \varphi(\alpha)-\varphi(0) \leq$ $\psi(\alpha)-\psi(0)$. Thus for $\alpha>0$,

$$
\frac{\varphi(\alpha)-\varphi(0)}{\alpha} \leq \frac{\psi(\alpha)-\psi(0)}{\alpha}
$$


and for $\alpha<0$,

$$
\frac{\varphi(\alpha)-\varphi(0)}{\alpha} \geq \frac{\psi(\alpha)-\psi(0)}{\alpha}
$$

Since $\varphi^{\prime}(0)$ and $\psi^{\prime}(0)$ exist, we have, by (3.2),

$$
\varphi^{\prime}(0)=\lim _{\alpha \rightarrow 0^{+}} \frac{\varphi(\alpha)-\varphi(0)}{\alpha} \leq \lim _{\alpha \rightarrow 0^{+}} \frac{\psi(\alpha)-\psi(0)}{\alpha}=\psi^{\prime}(0),
$$

and, by (3.3),

$$
\varphi^{\prime}(0)=\lim _{\alpha \rightarrow 0^{-}} \frac{\varphi(\alpha)-\varphi(0)}{\alpha} \geq \lim _{\alpha \rightarrow 0^{-}} \frac{\psi(\alpha)-\psi(0)}{\alpha}=\psi^{\prime}(0) .
$$

Thus $\varphi^{\prime}(0)=\psi^{\prime}(0)$ and (i) is proved.

To prove (ii), we consider the set $A=\left\{\alpha>0: \varphi^{\prime}(\alpha)=\psi^{\prime}(\alpha)\right\}$.

If $\inf \{\alpha \in A\}=0$, then there exists a sequence $\left\{\alpha_{n}\right\}_{n=1}^{\infty} \subset A$ so that $\lim _{n \rightarrow \infty} \alpha_{n}=0$. Since $\varphi^{\prime \prime}(0)$ and $\psi^{\prime \prime}(0)$ exist, and by (i), we obtain

$$
\varphi^{\prime \prime}(0)=\lim _{n \rightarrow \infty} \frac{\varphi^{\prime}\left(\alpha_{n}\right)-\varphi^{\prime}(0)}{\alpha_{n}}=\lim _{n \rightarrow \infty} \frac{\psi^{\prime}\left(\alpha_{n}\right)-\psi^{\prime}(0)}{\alpha_{n}}=\psi^{\prime \prime}(0)=0,
$$

so (ii) is proved.

If $\inf \{\alpha \in A\}>0$ (this includes the case that $A=\varnothing$ and then we say $\inf \{\alpha \in A\}=\infty>$ $0)$, then there exists $\varepsilon, 0<\varepsilon<\inf \{\alpha \in A\}$, so that $\varphi^{\prime}(\alpha) \neq \psi^{\prime}(\alpha)$ for all $\alpha \in(0, \varepsilon)$.

Let $h=\psi-\varphi$. Then $h(\alpha) \geq 0$ for all $\alpha \in \mathbb{R}, h(0)=0$, and $h^{\prime}(\alpha) \neq 0$ for all $\alpha \in(0, \varepsilon)$. Since $h^{\prime}$ satisfies the Darboux property, we get either

$$
h^{\prime}(\alpha)>0 \quad \forall \alpha \in(0, \varepsilon),
$$

or

$$
h^{\prime}(\alpha)<0 \quad \forall \alpha \in(0, \varepsilon) .
$$

But $h(0)=0$ and $h(\varepsilon) \geq 0$, so by the mean value theorem, there exists $\alpha_{0} \in(0, \varepsilon)$ so that $h^{\prime}\left(\alpha_{0}\right)=h(\varepsilon) / \varepsilon \geq 0$. Thus (3.7) has to hold. This implies that since $h^{\prime \prime}(0)$ exists, $h^{\prime \prime}(0) \geq$ 0 . This means that $0=\psi^{\prime \prime}(0) \geq \varphi^{\prime \prime}(0)$. Since $\varphi$ is convex, we also get $\varphi^{\prime \prime}(0) \geq 0$. Thus $\varphi^{\prime \prime}(0)=0$ and (ii) is proved.

To prove (iii), we denote $E_{1}=\left\{\alpha \in[0,1]: \varphi^{\prime \prime}(\alpha)\right.$ exists $\}$. Since $\varphi$ and $\psi$ are convex, $\mu\left(E_{1}\right)=1$. Without loss of generality, we can also assume that

$$
\varphi^{\prime \prime}(\alpha) \longrightarrow \infty \quad \text { as } \alpha \longrightarrow 0, \alpha \in E_{1} .
$$

Suppose, for contradiction, that there exists $C>0$ so that the set $E_{2}=\{\alpha \in[0,1]$ : $\psi^{\prime \prime}(\alpha)$ exists and $\left.\psi^{\prime \prime}(\alpha) \leq C\right\}$ has full measure. Let $E=E_{1} \cap E_{2}$. By (3.9), there exists $\varepsilon>0$ so that

$$
\varphi^{\prime \prime}(\alpha)>C \quad \text { for every } \alpha \in E \cap(0, \varepsilon) \text {. }
$$


Now consider the set $A=\left\{\alpha>0: \varphi^{\prime}(\alpha)=\psi^{\prime}(\alpha)\right\}$ similarly as we did in the proof of (ii). If inf $\{\alpha \in A\}=0$, then there exist $\alpha_{1}, \alpha_{2} \in(0, \varepsilon)$ so that $\alpha_{1} \neq \alpha_{2}$ and

$$
\varphi^{\prime}\left(\alpha_{1}\right)=\psi^{\prime}\left(\alpha_{1}\right), \quad \varphi^{\prime}\left(\alpha_{2}\right)=\psi^{\prime}\left(\alpha_{2}\right)
$$

But, since $\varphi^{\prime}$ is absolutely continuous on $[0,1]$, and by (3.10), we also have

$$
\varphi^{\prime}\left(\alpha_{1}\right)-\varphi^{\prime}\left(\alpha_{2}\right)=\int_{\alpha_{1}}^{\alpha_{2}} \varphi^{\prime \prime}(\alpha) d \alpha=\int_{\left[\alpha_{1}, \alpha_{2}\right] \cap E} \varphi^{\prime \prime}(\alpha) d \alpha>C\left(\alpha_{1}-\alpha_{2}\right)
$$

On the other hand, by the absolute continuity of $\psi^{\prime}$ on $[0,1]$ and the definition of $E_{2}$, we have

$$
\psi^{\prime}\left(\alpha_{1}\right)-\psi^{\prime}\left(\alpha_{2}\right)=\int_{\alpha_{1}}^{\alpha_{2}} \psi^{\prime \prime}(\alpha) d \alpha=\int_{\left[\alpha_{1}, \alpha_{2}\right] \cap E} \psi^{\prime \prime}(\alpha) d \alpha \leq C\left(\alpha_{1}-\alpha_{2}\right) .
$$

This is a contradiction since (3.11) implies that $\varphi^{\prime}\left(\alpha_{1}\right)-\varphi^{\prime}\left(\alpha_{2}\right)=\psi^{\prime}\left(\alpha_{1}\right)-\psi^{\prime}\left(\alpha_{2}\right)$.

Now we consider the case that $\inf \{\alpha \in A\} \neq 0$, that is, $\inf \{\alpha \in A\}>0$ (this, as in (ii), includes the possibility that $A=\varnothing$ in which case we say that inf $\{\alpha \in A\}=\infty)$. We showed in the proof of (ii) (cf. (3.7)) that in this case, there exists $\varepsilon_{1}, 0<\varepsilon_{1}<\inf \{\alpha \in A\}$, so that

$$
\psi^{\prime}(\alpha)>\varphi^{\prime}(\alpha) \quad \forall \alpha \in\left(0, \varepsilon_{1}\right)
$$

By (i), $\varphi^{\prime}(0)=\psi^{\prime}(0)$. Let $\alpha_{0} \in(0, \varepsilon) \cap\left(0, \varepsilon_{1}\right)$. Then, similarly as in the previous case, since $\varphi^{\prime}$ is absolutely continuous on $[0,1]$, by $(3.10)$, we obtain

$$
\varphi^{\prime}\left(\alpha_{0}\right)-\varphi^{\prime}(0)=\int_{0}^{\alpha_{0}} \varphi^{\prime \prime}(\alpha) d \alpha=\int_{\left[0, \alpha_{0}\right] \cap E} \varphi^{\prime \prime}(\alpha) d \alpha>C \alpha_{0}
$$

On the other hand, again by the absolute continuity of $\psi^{\prime}$ and the definition of $E_{2}$,

$$
\psi^{\prime}\left(\alpha_{0}\right)-\psi^{\prime}(0)=\int_{0}^{\alpha_{0}} \psi^{\prime \prime}(\alpha) d \alpha=\int_{\left[0, \alpha_{0}\right] \cap E_{2}} \psi^{\prime \prime}(\alpha) d \alpha \leq C \alpha_{0}
$$

Thus $\psi^{\prime}\left(\alpha_{0}\right)<\varphi^{\prime}\left(\alpha_{0}\right)$, which contradicts (3.14) and ends the proof of (iii).

We are now ready for our main results.

Theorem 3.2. Let $M$ be an Orlicz function with $M \in \Delta_{2+}$ and let $P$ be a contractive projection on the real Orlicz sequence space $\ell_{M}$ equipped with the Luxemburg norm. Then the 
following hold:

(a) if $M \in A C^{2}, M^{\prime \prime}(0)=0$, and $M^{\prime \prime}(t)>0$ for all $t>0$, then $P$ is semi-band preserving;

(b) if $M \in A C^{1}$ near zero, $M^{\prime \prime}$ is continuous on $(0, \infty)$, and $\lim _{t \rightarrow 0} M^{\prime \prime}(t)=\infty$, then $P$ is semi-containment preserving.

Proof. Since bounded functions with finite supports are linearly dense in $\ell_{M}$, to show that $P$ is semi-band preserving or semi-containment preserving, respectively, it is enough to verify that (2.14) or (2.15), respectively, are satisfied with the additional assumption that $g$ is a bounded function and $\operatorname{card}(\operatorname{supp} g)<\infty$.

For any functions $f, g \in \ell_{M}$, we define

$$
\psi(\alpha)=\|P f+\alpha g\|_{M}, \quad \varphi(\alpha)=\|P f+\alpha P g\|_{M}
$$

for all $\alpha \in \mathbb{R}$. Then $\varphi$ and $\psi$ are convex functions and $\psi(0)=\|P f\|=\varphi(0)$. Moreover, by Theorem 2.6, in both cases (a) and (b), $\varphi$ and $\psi$ are differentiable everywhere. Since $P$ is a contractive projection, we also get $\varphi(\alpha) \leq \psi(\alpha)$ for all $\alpha \in \mathbb{R}$.

Now to prove (a), assume that $\operatorname{card}(\operatorname{supp} g)<\infty$ and $\operatorname{supp}(g) \cap \operatorname{supp}(P f)=\varnothing$. Since $M \in A C^{2}$, by Theorem 2.6, $\varphi^{\prime \prime}(0)$ and $\psi^{\prime \prime}(0)$ exist. By Proposition 2.9(a), we get $\psi^{\prime}(0)=$ 0 and $\psi^{\prime \prime}(0)=0$. Hence by Lemma 3.1(i) and (ii), $\varphi^{\prime}(0)=0$ and $\varphi^{\prime \prime}(0)=0$. Thus, by Proposition 2.9(b), we get that $P f$ and $P g$ have disjoint supports, which proves that $P$ is semi-band preserving.

To prove (b), assume, for contradiction, that there exist $f, g \in \ell_{M}$ so that card(supp $g$ ) < $\infty, \operatorname{supp}(g) \subseteq \operatorname{supp}(P f)$, and $\operatorname{supp}(P g) \nsubseteq \operatorname{supp}(P f)$. Note that since $M \in A C^{1}$, by Theorem 2.6, functions $\varphi$ and $\psi$ are differentiable everywhere, $\varphi^{\prime}, \psi^{\prime}$ are absolutely continuous, and $\varphi^{\prime \prime}, \psi^{\prime \prime}$ exist almost everywhere. Further, by Proposition 2.10(b), there exists a subset $E$ of $[0,1]$ of full measure and $C_{0}>0$ such that for all $\alpha \in E$,

$$
\psi^{\prime \prime}(\alpha) \leq C_{0}
$$

On the other hand, by Proposition 2.10(b), $\psi^{\prime \prime}(\alpha) \rightarrow \infty$, as $\alpha \rightarrow 0$ along a subset of $[0,1]$ of full measure. Hence, by Lemma 3.1(iii) for every $C>0,(3.1)$ holds. This contradicts (3.18) and ends the proof of part (b).

As a consequence, we obtain the characterization of contractive projections in Orlicz sequence spaces.

TheOREM 3.3. Let $M$ be an Orlicz function with $M \in \Delta_{2+}$ and satisfying one of the following two conditions:

(i) $M \in A C^{2}, M^{\prime \prime}(0)=0$, and $M^{\prime \prime}(t)>0$ for all $t>0$; or

(ii) $M \in A C^{1}, M^{\prime \prime}$ is continuous on $(0, \infty)$, and $\lim _{t \rightarrow 0} M^{\prime \prime}(t)=\infty$.

Let $P$ be a contractive projection on the real Orlicz sequence space $\ell_{M}$ equipped with the Luxemburg norm. Then $P$ is an averaging projection, that is, there exist mutually disjoint elements $\left\{u_{j}\right\}_{j \in J}$ in $\ell_{M}$ and functionals $\left\{u_{j}^{*}\right\}_{j \in J}$ in $\left(\ell_{M}\right)^{*}$ so that $u_{j}^{*}\left(u_{k}\right)=0$ if $j \neq k$, $u_{j}^{*}\left(u_{j}\right)=1$ for all $j \in J$, and for each $f \in \ell_{M}$, (2.13) holds. Moreover, one of the following 
three possibilities holds:

(1) $\operatorname{card}\left(\operatorname{supp} u_{j}\right)<\infty$ for each $j \in J$, and $\left|\left(u_{j}\right)_{k}\right|=\left|\left(u_{j}\right)_{l}\right|$ for each $k, l \in \operatorname{supp}\left(u_{j}\right), j \in$ J. $\left(\right.$ Here $\left.u_{j}=\sum_{k \in \operatorname{supp} u_{j}}\left(u_{j}\right)_{k} e_{k}\right)$;

(2) there exist $p \in(1, \infty) \backslash\{2\}$ and $C \in \mathbb{R}$ so that $M(t)=C t^{p}$ for all $t \leq \sup _{j \in J}\left\|u_{j}\right\|_{\infty}(\leq$ $\infty)$; or

(3) there exist $p \in(1, \infty) \backslash\{2\}$ and constants $C_{1}, C_{2}, \gamma>0$ so that $C_{2} t^{p} \leq M(t) \leq C_{1} t^{p}$ for all $t \leq \sup _{j \in J}\left\|u_{j}\right\|_{\infty}(\leq \infty),\left\|u_{j}\right\|_{\infty}<\infty$ for all $j \in J$, and for all $j \in J$ and $k \in$ $\operatorname{supp}\left(u_{j}\right)$,

$$
\left|\left(u_{j}\right)_{k}\right| \in\left\{\gamma^{m} \cdot\left\|u_{j}\right\|_{\infty}: m \in \mathbb{Z}\right\}
$$

Proof. Note first that either condition (i) or (ii) implies that $\ell_{M}$ is smooth. Since $M \in \Delta_{2}$, $\ell_{M}$ is strictly monotone. Hence the fact that $P$ is an averaging projection follows immediately from Theorems 2.8 and 3.2.

The moreover part follows directly from Theorem 2.13. Indeed, since the elements $\left\{u_{j}\right\}_{j \in J}$ are mutually disjoint, for any $j_{1}, j_{2} \in J$ and any $f \in \ell_{M}$, we have

$$
\left\|u_{j_{1}}^{*}(f) u_{j_{1}}+u_{j_{2}}^{*}(f) u_{j_{2}}\right\| \leq\left\|\sum_{j \in J} u_{j}^{*}(f) u_{j}\right\|=\|P f\| \leq\|f\| .
$$

Thus the projection $Q: \ell_{M} \rightarrow \operatorname{span}\left\{u_{j_{1}}, u_{j_{2}}\right\}$ defined by $Q f=u_{j_{1}}^{*}(f) u_{j_{1}}+u_{j_{2}}^{*}(f) u_{j_{2}}$ has $\|Q\|=1$. Thus, by Theorem 2.13, conditions (1), (2), and (3) in the statement of Theorem 3.3 are satisfied.

By duality, we also obtain the description of contractive projections in real Orlicz sequence spaces equipped with the Orlicz norm.

Corollary 3.4. Let $M$ be an Orlicz function with $M \in \Delta_{2}, M^{*} \in \Delta_{2+}$, satisfying one of the following two conditions:

(i*) $M^{*} \in A C^{2}, M^{\prime \prime}$ is continuous on $(0, \infty), M^{\prime \prime}(t)>0$ for all $t>0$, and $\lim _{t \rightarrow 0} M^{\prime \prime}(t)=$ $\infty$; or

(ii*) $M^{*} \in A C^{1}, M^{\prime \prime}$ is continuous on $[0, \infty), M^{\prime \prime}(t)>0$ for all $t>0$, and $M^{\prime \prime}(0)=0$.

Let $P$ be a contractive projection on the real Orlicz sequence space $\ell_{M}$ equipped with the Orlicz norm. Then $P$ has the form described in Theorem 3.3.

Proof. This follows from Theorem 3.3 by duality. Indeed, since $M \in \Delta_{2}$, by (2.7) we have $\left(\ell_{M},\|\cdot\|_{M}^{O}\right)^{*}=\left(\ell_{M^{*}},\|\cdot\|_{M^{*}}\right)$ and the dual projection $P^{*}$ is contractive in $\ell_{M^{*}}$ equipped with the Luxemburg norm. Further, either of the conditions (i*) or (ii*) implies that $M^{*}$ is smooth, so the only thing that needs to be verified is that condition ( $\left.i^{*}\right)$ implies that $M^{*}$ satisfies condition (i) and condition (ii*) implies that $M^{*}$ satisfies condition (ii) of Theorem 3.3.

For that, note that by the definition of the complementary function $M^{*}$ and since in either case (i*) or (ii*), $M^{\prime \prime}(t)>0$ for $t>0$, we have, for all $t>0$,

$$
\left(M^{*}\right)^{\prime \prime}(t)=\frac{1}{M^{\prime \prime}\left(\left(M^{*}\right)^{\prime}(t)\right)} .
$$


Since $M^{\prime \prime}$ and $\left(M^{*}\right)^{\prime}$ are both continuous on $(0, \infty)$ in either case $\left(\mathrm{i}^{*}\right)$ or $\left(\mathrm{ii}^{*}\right)$, we conclude that also $\left(M^{*}\right)^{\prime \prime}$ is continuous on $(0, \infty)$ and $\left(M^{*}\right)^{\prime \prime}(t)>0$ for all $t>0$.

Moreover, since $\lim _{t \rightarrow 0}\left(M^{*}\right)^{\prime}(t)=\left(M^{*}\right)^{\prime}(0)=0$, we have in case $\left(\mathrm{i}^{*}\right)$

$$
\lim _{t \rightarrow 0}\left(M^{*}\right)^{\prime \prime}(t)=\lim _{t \rightarrow 0} \frac{1}{M^{\prime \prime}\left(\left(M^{*}\right)^{\prime}(t)\right)}=\lim _{s \rightarrow 0} \frac{1}{M^{\prime \prime}(s)}=0 .
$$

It is not difficult to check that this implies that $\left(M^{*}\right)^{\prime \prime}(0)=0$. Therefore, condition (i) is implied by $\left(\mathrm{i}^{*}\right)$.

Similarly, in case (ii*), we have

$$
\lim _{t \rightarrow 0}\left(M^{*}\right)^{\prime \prime}(t)=\lim _{t \rightarrow 0} \frac{1}{M^{\prime \prime}\left(\left(M^{*}\right)^{\prime}(t)\right)}=\lim _{s \rightarrow 0} \frac{1}{M^{\prime \prime}(s)}=\infty .
$$

So condition (ii) is implied by (ii*).

Hence, by Theorem 3.3, in either case ( $\left.\mathrm{i}^{*}\right)$ or (ii*), $P^{*}$, and thus also $P$, have the form (2.13) and the conditions (1), (2), and (3) from Theorem 3.3 hold.

Remark 3.5. We do not know whether the assumption about smoothness of $M$ is necessary for Theorem 3.3 and Corollary 3.4 to hold or not. We suspect that, similarly as in the complex case, smoothness of $M$ should not be necessary.

However it is clear that some assumption about a behavior of $M^{\prime \prime}$ near zero is necessary. Indeed in [16, Example 3], we showed that if $a \in(\sqrt{2 / 3}, 1)$ and

$$
M_{a}(t)= \begin{cases}t^{2} & \text { if } 0 \leq t \leq a \\ (1+a) t-a & \text { if } a \leq t \leq 1\end{cases}
$$

then the real or complex four-dimensional Orlicz space $\ell_{M_{a}}^{4}$ equipped with either the Luxemburg or the Orlicz norm contains a two-dimensional one-complemented isometric copy of $\ell_{2}^{2}$ which cannot be spanned by a family of disjoint vectors from $\ell_{M_{a}}^{4}$. It is not difficult to adjust this example so that if $a$ is any positive number, then the real or complex Orlicz space $\ell_{M_{a}}$ (of infinite dimension) contains a two-dimensional one-complemented isometric copy of $\ell_{2}^{2}$ which cannot be spanned by a family of disjoint vectors from $\ell_{M_{a}}$.

It would be interesting to characterize what condition on $M$ is equivalent to the fact that $\ell_{M}$ (complex or real) does not contain a two-dimensional one-complemented isometric copy of $\ell_{2}^{2}$ (which cannot be spanned by a family of disjoint vectors from $\ell_{M}$ ). Either of the conditions (i), (ii), (i*), or (ii*) is clearly sufficient, but they all involve smoothness. We conjecture that the right condition is that for all $a>0$, the function $M(t) / t^{2}$ is not constant on the interval $(0, a)$.

\section{Acknowledgments}

The author wishes to thank the organizers of the National Science Foundation Workshop on Linear Analysis and Probability at Texas A\&M University for inviting her to participate in this workshop in Summer 2001, when part of this research was done. The author also acknowledges the partial support from the National Science Foundation and Association for Women in Mathematics Travel Grant in 2001. 


\section{References}

[1] T. Andô, Contractive projections in $L_{p}$ spaces, Pacific J. Math. 17 (1966), 391-405.

[2] J. Blatter and E. W. Cheney, Minimal projections on hyperplanes in sequence spaces, Ann. Mat. Pura Appl. (4) 101 (1974), 215-227.

[3] S. Chen, Geometry of Orlicz spaces, Dissertationes Math. (Rozprawy Mat.) 356 (1996), 1-204.

[4] S. T. Chen, Smoothness of Orlicz spaces, Comment. Math. Prace Mat. 27 (1987), no. 1, 49-58.

[5] E. W. Cheney and K. H. Price, Minimal projections, Approximation Theory (Proc. Sympos., Lancaster, 1969), Academic Press, London, 1970, pp. 261-289.

[6] R. G. Douglas, Contractive projections on an $L_{1}$ space, Pacific J. Math. 15 (1965), 443-462.

[7] J. E. Jamison, A. Kamińska, and G. Lewicki, One complemented subspaces of Musielak-Orlicz sequence spaces, preprint, 2001, http://www.im.uj.edu.pl/preprinty/\#pre2001.

[8] M. A. Krasnosel'skiĭ and Ja. B. Rutickiĭ, Convex Functions and Orlicz Spaces, P. Noordhoff, Groningen, 1961.

[9] W. Kurc, Strictly and uniformly monotone sequential Musielak-Orlicz spaces, Collect. Math. 50 (1999), no. 1, 1-17.

[10] K. Lindberg, On subspaces of Orlicz sequence spaces, Studia Math. 45 (1973), 119-146.

[11] J. Lindenstrauss and L. Tzafriri, Classical Banach Spaces. I. Sequence Spaces, Ergebnisse der Mathematik und ihrer Grenzgebiete, vol. 92, Springer-Verlag, Berlin, 1977.

[12] R. P. Maleev and S. L. Troyanski, Smooth norms in Orlicz spaces, Canad. Math. Bull. 34 (1991), no. $1,74-82$.

[13] W. Matuszewska and W. Orlicz, On certain properties of $\varphi$-functions, Bull. Acad. Polon. Sci. Sér. Sci. Math. Astronom. Phys. 8 (1960), 439-443.

[14] W. Odyniec and G. Lewicki, Minimal Projections in Banach Spaces. Problems of Existence and Uniqueness and Their Application, Lecture Notes in Mathematics, vol. 1449, SpringerVerlag, Berlin, 1990.

[15] B. Randrianantoanina, A disjointness type property of conditional expectation operators, to appear in Colloq. Math.

[16] , 1-complemented subspaces of spaces with 1-unconditional bases, Canad. J. Math. 49 (1997), no. 6, 1242-1264.

[17] One-complemented subspaces of real sequence spaces, Results Math. 33 (1998), no. 1-2, $139-154$.

[18] Injective isometries in Orlicz spaces, Function Spaces (Edwardsville, Ill, 1998), Contemp. Math., vol. 232, Amer. Math. Soc., Rhode Island, 1999, pp. 269-287.

[19]__ Norm-one projections in Banach spaces, Taiwanese J. Math. 5 (2001), no. 1, 35-95.

[20] M. M. Rao and Z. D. Ren, Theory of Orlicz Spaces, Monographs and Textbooks in Pure and Applied Mathematics, vol. 146, Marcel Dekker, New York, 1991.

Beata Randrianantoanina: Department of Mathematics and Statistics, Miami University, Oxford, $\mathrm{OH} 45056$, USA

E-mail address: randrib@muohio.edu 


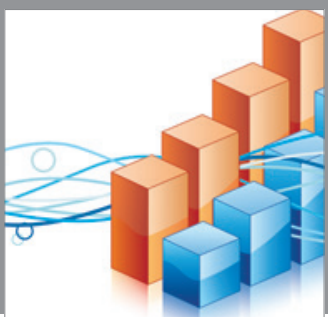

Advances in

Operations Research

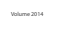

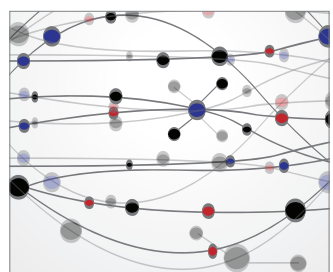

\section{The Scientific} World Journal
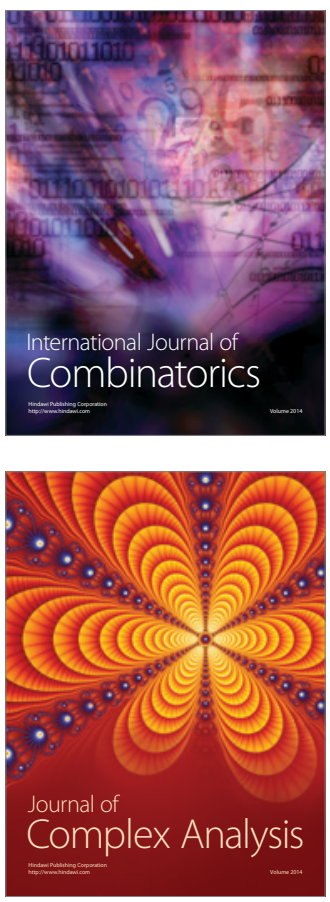

International Journal of

Mathematics and

Mathematical

Sciences


Journal of

Applied Mathematics
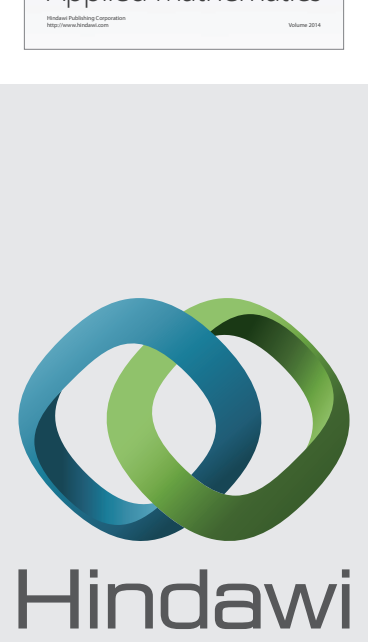

Submit your manuscripts at http://www.hindawi.com
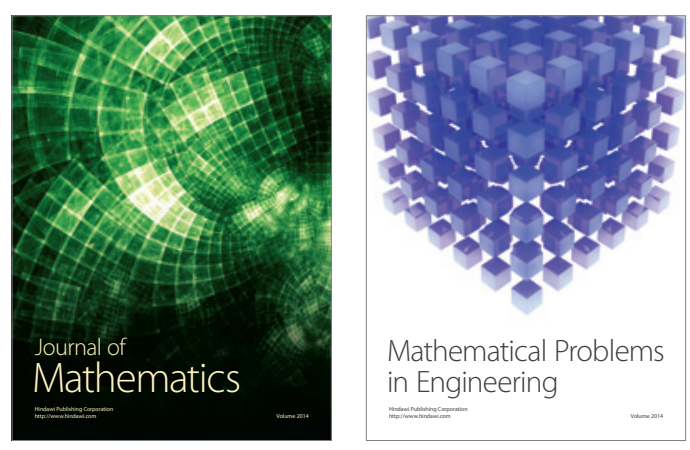

Mathematical Problems in Engineering
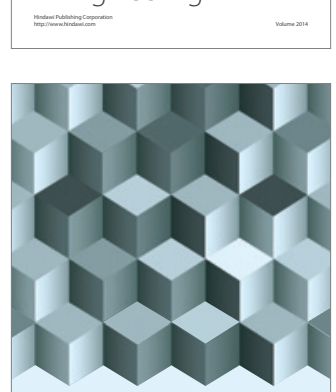

Journal of

Function Spaces
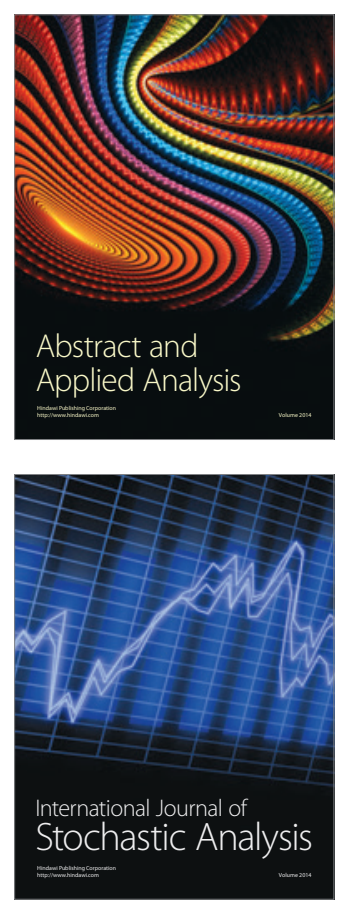

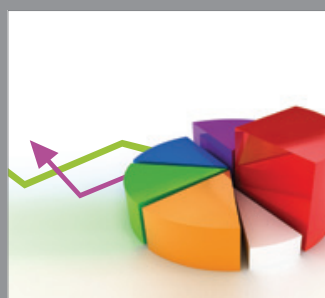

ournal of

Probability and Statistics

Promensencen
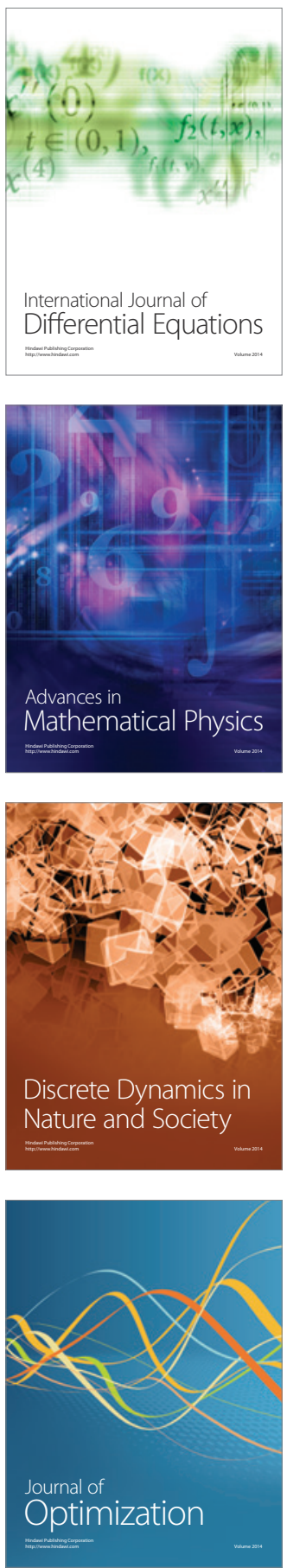